\title{
Naloxone should remain the appropriate antidote to treat opioid overdose
}

\author{
Bruno Mégarbane ${ }^{1,2^{*}}$ (D) Lucie Chevillard ${ }^{2}$ and Dominique Vodovar ${ }^{2,3}$
}

To the Editor,

In a randomized trial, Zamani et al. assessed the greater effectiveness and safety of buprenorphine versus naloxone to reverse methadone overdose-related respiratory depression [1]. We congratulate the authors. However, several issues may limit the accuracy of their observations and recommendation to unselectively treat opioid overdoses with buprenorphine.

Usually, opioid overdose is identified in patients presenting stupor or coma (i.e., Glasgow coma score [GCS] $\leq 12$ ), respiratory rate $[R R] \leq 12 / \mathrm{min}$ and miosis) [2]. Zamani's patients were mildly poisoned with mean/median (not clear) RR of $12-15 / \mathrm{min}$ and GCS of $13-15$. Therefore, the elevated naloxone-induced withdrawal rate clearly resulted from excessive dosage and/or inadequate titration aiming to obtain "complete arousal to normal consciousness" in mildly overdosed opioiddependent patients. Opioid-tolerant patients frequently respond to low naloxone doses, sufficient to restore breathing without provoking withdrawal [2]. Clinicians incorrectly assume that the naloxone dose required to restore respiration correlates with severity. Naloxone should be injected at $0.04 \mathrm{mg}$ bolus and titrated $/ 2 \mathrm{~min}$ upward to effect (i.e., $R R \geq 15 / \mathrm{min}$ ).

Naloxone-attributed complications were surprisingly frequent, not reflecting our practice, especially since patients with cardiovascular morbidities, co-ingestions, aspiration, and need for immediate intubation were excluded. ARDS onset in 15\% of naloxone-treated

This comment refers to the article available at https://doi.org/10.1186/ s13054-020-2740-y

* Correspondence: bruno.megarbane@lrb.aphp.fr

'Department of Medical and Toxicological Critical Care, Lariboisière-Fernand

Widal Hospital, Federation of Toxicology APHP, Paris University, 2 Rue

Ambroise Paré, 75010 Paris, France

${ }^{2}$ INSERM UMRS-1144, Paris University, Paris, France

Full list of author information is available at the end of the article patients without prior aspiration is unusual. Naloxone has been mistakenly implicated as a cause of pulmonary edema [2]. Using optimal regimens allows the restoration of oxygenation and prevents the postulated sympathetic surge that triggers pulmonary edema after apnea reversal.

In Fig. 2, 13 apnea episodes were reported in the naloxone group, mostly reversed by naloxone boluses. Management of the 11 apnea episodes in the buprenorphine groups was not provided since only five patients were intubated. Did the patients receive buprenorphine boluses or continuous infusion?

Intravenous buprenorphine should be used cautiously in opioid users who, unlike the Iranian experience, mostly co-abuse benzodiazepines and/or ethanol [3]. Drug-drug interactions and intravenous buprenorphine administration are the two well-recognized circumstances that impair buprenorphine-related ceiling effects, worsening ventilation consequently, as demonstrated experimentally $[4,5]$. Because naloxone is lacking agonist activity on the mu-opioid receptor, no such risk exists. Additionally, in non-methadone abusers, buprenorphine may compete with the existing opioid on the mu-opioid receptor and, due to higher binding affinity, produce late withdrawal if the opioid exhibits shorter elimination half-life.

Therefore, unless convincing data are produced, naloxone should remain the legitimate antidote to reverse opioid toxicity. Concerns that naloxone may harm opioid-dependent patients are unfounded [2]. With adequate titration, signs of naloxone-induced abstinence are unpleasant but never life-threatening. 


\title{
Authors' response
}

\author{
Nasim Zamani ${ }^{1,2,3}$, Nicholas A. Buckley ${ }^{4}$ and Hossein Hassanian-Moghaddam ${ }^{1,2}$ \\ ${ }^{1}$ Social Determinant of Health Research Center, Shahid Beheshti University of Medical Sciences, Tehran, Iran \\ ${ }^{2}$ Department of Clinical Toxicology, Loghman Hakim Hospital, Shahid Beheshti University of Medical Sciences, \\ South Karegar Street, Tehran, Iran \\ ${ }^{3}$ Toxicological Research Center, Shahid Beheshti University of Medical Sciences, Tehran, Iran \\ ${ }^{4}$ Pharmacology, Faculty of Medicine and Health, University of Sydney, Sydney, Australia
}

To the Editor,

The comments mentioned are a reflection of the opinions of three clinicians working in a Paris ICU [1]. That is a setting typically many hours post-emergency resuscitation, and many of the complications with naloxone may fall outside their remit. Iran has a 100-fold higher rate of fatal/ non-fatal methadone overdose than France. The reported rate of methadone-related hospitalization was just 5.4 per 1,000,000 French inhabitants in 2017 [6]. Around 31 per 1,000,000 people died in Tehran due to methadone overdose in 2015, and this would be just a fraction of the total overdoses [1,7].

It is not the lack of experience that creates problems with managing respiratory depression without precipitating in withdrawal using titrated naloxone [1]. Methadone overdose is inherently difficult to manage with naloxone; it has a long and variable half-life with a resulting wide range of concentrations [7]. As seen in our article, the range of doses and durations of naloxone required both vary more than ten-fold. This contrasted with the simple (but more effective) buprenorphine dosing.

There is published evidence on the complications of naloxone particularly in higher doses [8]. A US study reported pulmonary complications in $26.5 \%$ of 1831 patients. Those receiving doses $>4.4 \mathrm{mg}$ had a $46 \%$ complication rate [7]. Due to the long half-life of methadone, most of our patients required such higher doses of naloxone to overcome opioid effects.

We reported GCS at the time of randomization, but this often reflected a preceding dose of naloxone in the prehospital setting. Evidence for severe toxicity requiring effective reversal is seen in rates of apnea (22\%), intubation (30\%), prolonged sedation (33\%), and ARDS (15\%). ARDS may be secondary to further apnea and aspiration or even as a result of higher doses of naloxone infusion [8]. Further, $15 \%$ of those treated with naloxone died. In terms of apnea reversal, in each group, apnea was reversed by naloxone or buprenorphine after hospitalization accordingly [1].

Megarbane et al. also raise two theoretical points based on rat studies. We believe the theory also indicates concerns about precipitating withdrawal and interacting drugs and alcohol apply to an even greater extent for naloxone. In contrast, using sublingual buprenorphine in the ED is beneficial in starting the process of buprenorphine maintenance therapy and thus in harm reduction and reducing further overdoses and deaths [9]. Further evidence from other settings are definitely needed to withdraw more robust results.

Yours truly,

Nasim Zamani, Nick A Buckley, and

Hossein Hassanian-Moghaddam.

\section{Acknowledgements}

The authors would like to acknowledge Mrs. Alison Good, Scotland, UK, for her helpful review of this manuscript.

Authors' contributions

All authors drafted, read, and approved the final manuscript.

\section{Funding}

None

Availability of data and materials

Not applicable

Ethics approval and consent to participate

Not applicable

Consent for publication

Not applicable

Competing interests

The authors declare that they have no competing interests.

\section{Author details}

'Department of Medical and Toxicological Critical Care, Lariboisière-Fernand Widal Hospital, Federation of Toxicology APHP, Paris University, 2 Rue Ambroise Paré, 75010 Paris, France. 'INSERM UMRS-1144, Paris University, Paris, France. ${ }^{3}$ Poison Control Center of Paris, Lariboisière-Fernand Widal Hospital, Federation of Toxicology APHP, Paris University, Paris, France.

Received: 28 February 2020 Accepted: 17 March 2020

Published online: 28 April 2020

\section{References}

1. Zamani N, Buckley NA, Hassanian-Moghaddam H. Buprenorphine to reverse respiratory depression from methadone overdose in opioid-dependent patients: a prospective randomized trial. Crit Care. 2020;24:44. https://doi. org/10.1186/s13054-020-2740-y.

2. Boyer EW. Management of opioid analgesic overdose. N Engl J Med. 2012; 367:146-55.

3. Mégarbane B, Buisine A, Jacobs F, Résière D, Chevillard L, Vicaut E, Baud FJ. Prospective comparative assessment of buprenorphine overdose with heroin and methadone: clinical characteristics and response to antidotal treatment. J Subst Abus Treat. 2010;38:403-7. 
4. Cohier C, Chevillard L, Salle S, Risède P, Roussel O, Mégarbane B. Editor's highlight: neurorespiratory effects of buprenorphine and ethanol in combination: a mechanistic study of drug-drug interactions in the rat. Toxicol Sci. 2017:155:389-99.

5. Cohier C, Chevillard L, Risède P, Roussel O, Mégarbane B. Respiratory effects of buprenorphine/naloxone alone and in combination with diazepam in naive and tolerant rats. Toxicol Lett. 2014;228:75-84.

6. Chenaf C, Kaboré JL, Delorme J, et al. Prescription opioid analgesic use in France: trends and impact on morbidity-mortality. Eur J Pain. 2019;23(1): 124-34. https://doi.org/10.1002/ejp.1291.

7. Akhgari M, Amini-Shirazi N, Iravani FS. Forensic toxicology perspectives of methadone-associated deaths in Tehran, Iran, a 7-year overview. Basic Clin Pharmacol Toxicol. 2018;122(4):436-41.

8. Farkas $A$, et al. Pulmonary complications of opioid overdose treated with naloxone. Ann Emerg Med. 2020;75(1):39-48.

9. Fox L, Nelson LS. Emergency department initiation of buprenorphine for opioid use disorder: current status, and future potential. CNS Drugs. 2019; 33(12):1147-54.

\section{Publisher's Note}

Springer Nature remains neutral with regard to jurisdictional claims in published maps and institutional affiliations. 